\title{
Transverse Spin Effects In Diffractive Hadron Leptoproduction
}

\author{
S.V.Goloskokov 中 \\ Bogoliubov Laboratory of Theoretical Physics, \\ Joint Institute for Nuclear Research. \\ Dubna 141980, Moscow region, Russia.
}

\begin{abstract}
We consider double spin asymmetries for longitudinally polarized leptons and transversely polarized protons in diffractive vector meson and $Q \bar{Q}$ production at high energies within the two-gluon model. The asymmetry predicted for meson production is quite small. The $A_{l T}$ asymmetry for $Q \bar{Q}$ production contains two independent terms which are large and can be used to obtain information on the polarized gluon distributions in the proton.
\end{abstract}

\footnotetext{
${ }^{1}$ Email: goloskkv@thsun1.jinr.ru
} 


\section{Introduction}

Study of the hadron structure is a fundamental problem of modern physics. One of the important objects here is parton distributions in a nucleon. The cross section of inclusive hadron production is expressed in terms of ordinary parton distributions where partons have the same momenta. More general structures in a nucleon can be studied in deeply virtual Compton scattering or in diffractive hadron leptoproduction. Really, the kinematics of there processes requires a nonzero longitudinal momentum $\zeta p$ carried by a two-parton system. As a result, the parton momenta cannot be equal, and such reactions are expressed in terms of skewed parton distributions (SPD) [1, 2]. The factorization of the amplitude of diffractive hadron production into a hard subprocess and a soft proton matrix element- SPD has been shown in [3]. The diffractive charm $Q \bar{Q}$ production and $J / \Psi$ production are determined by the gluon $\operatorname{SPD} \mathcal{F}_{\zeta}(x)$, because the charm component in the proton is small. The processes with light quarks are predominated at small Bjorken $x \leq 0.1$ by the Pomeron exchange which can be associated with a two-gluon state [4. Both quark and gluon SPD will contribute here for $x>0.1$.

Sensitivity of diffractive lepto and photoproduction to the gluon density in the proton gives an excellent tool to test these structure functions. Intensive experimental study of diffractive processes were performed in DESY (see e.g. [5, 6, 7, 8] and references therein). The longitudinal double spin asymmetry in vector meson production has been analysed in [9]. Theoretical investigation of the diffractive vector meson production was conducted on the basis of different models where sensitivity of the experimental observables to polarized parton distributions was studied. Within the two- gluon exchange model, the typical scale $\bar{Q}^{2}=\left(Q^{2}+M_{V}^{2}\right) / 4$ was found for vector mesons production [10, 11]. The cross sections of light and heavy meson production plotted versus this variable looks similar [12]. In papers [13, 14, 15] the cross section for longitudinal and transverse photon polarization was analyzed. It was shown that a longitudinally polarized photon gives a predominat contribution to the cross section for $Q^{2} \rightarrow \infty$. The cross section with transverse photon polarization is suppressed as a power of $Q$. Investigation of the vector meson production within the SPD approach was performed by many authors (see e.g [16, 17]). Within the SPS approach, one can study simultaneously the imaginary and real parts of diffractive amplitudes. In paper [18], the double spin asymmetry for longitudinal photon and proton polarization in $J / \Psi$ production was estimated. The contribution with transversely polarized photons and a vector meson is important for the spin observable like $A_{l l}$ asymmetry. Unfortunately, for the light meson production, this higher twist transition amplitude is not well defined because of the present infrared singularities [19. 
Another possibility to study SPD in the proton is based on the quark pair leptoproduction. Theoretical analysis of the diffractive $Q \bar{Q}$ production which can be observed as two jet events in lepton-proton interaction was carried out e.g. in [20, 21, 22, 23]. It was shown that the cross sections of diffractive quark- antiquark production are expressed in terms of gluon distributions as in the case of vector meson production. However, the scale variable in the structure function is here determined by the transverse momentum of a produced quark. Spin effects in diffractively produced quark-antiquark pair for longitudinally polarized lepton and proton was discussed in [24] where the diffractive contribution to the $g_{1}$ structure function was calculated.

Thus, the diffractive reactions should play a key role in the study of the gluon structure of the proton at small $x$. In the case of polarized particles, the spin-dependent gluon distributions can be investigated. Previously, spin asymmetries for longitudinally polarized particles were mainly analyzed. In future, it will be an excellent possibility to study spin effects with transversely polarized target at HERMES. Numerous proposals for possible experiments with this target were discussed in 25]. Such experiments should shed light on the polarized parton distributions which are responsible on the transverse spin effects in the hadron.

In this paper we consider double spin asymmetries for longitudinally polarized leptons and transversely polarized protons in diffractive vector meson and $Q \bar{Q}$ production at high energies. Some preliminary results in this field were published in [26]. The two-gluon exchange model with the spin-dependent $g g$-proton coupling is used. This means that our results should be applicable for reactions with heavy quarks which are determined by the gluons exchange. For processed with light quarks our predictions should be valid at small $x$ ( $x \leq 0.1$ e.g. $)$. The cross section of hadrons leptoproduction can be decomposed into the leptonic and hadronic tensors and the amplitude of hadron production through the $\gamma^{\star} g g$ transition to the vector meson or $Q \bar{Q}$ states. After describing the kinematics of the process in Sect. 2, we analyze the structure of the leptonic and hadronic tensors in Sect. 3. In Sect. 4 , we calculate the polarized cross section of vector meson leptoproduction. Connection of the two-gluon approach with skewed gluon distributions is discussed, too. Similar results for diffractive $Q \bar{Q}$ production are presented in Sect. 5. The numerical results for the diffractive vector meson and production at HERMES and COMPASS energies and our prediction for the $A_{l T}$ asymmetry can be found in Sects 6 and 7 . We finish with concluding remarks in Sect. 8. 


\section{Kinematics of Diffractive Hadron Leptoproduction}

Let us study the diffractive hadron production in lepton-proton reactions

$$
l+p \rightarrow l+p+H
$$

at high energies in a lepton-proton system. The hadron state $H$ in this reaction can contain a vector meson or a $Q \bar{Q}$ system which can be detected as two final jets. The reaction (11) can be described in terms of the kinematic variables which are defined as follows:

$$
\begin{array}{r}
q^{2}=\left(l-l^{\prime}\right)^{2}=-Q^{2}, t=r_{P}^{2}=\left(p-p^{\prime}\right)^{2}, \\
y=\frac{p \cdot q}{l \cdot p}, x=\frac{Q^{2}}{2 p \cdot q}, x_{P}=\frac{q \cdot\left(p-p^{\prime}\right)}{q \cdot p}, \beta=\frac{x}{x_{P}}
\end{array}
$$

where $l, l^{\prime}$ and $p, p^{\prime}$ are the initial and final lepton and proton momenta, respectively, $Q^{2}$ is the photon virtuality, and $r_{P}$ is the momentum carried by the Pomeron. The variable $\beta$ is used in $Q \bar{Q}$ production. In this case the effective mass of a produced quark system is equal to $M_{X}^{2}=\left(q+r_{P}\right)^{2}$ and can be quite large. The new variable $\beta=x / x_{P} \sim Q^{2} /\left(M_{X}^{2}+Q^{2}\right)$ which appears in this case can vary from 0 to 1 . For diffractive vector meson production, $M_{X}^{2}=M_{V}^{2}$ and $\beta \sim 1$ for large $Q^{2}$. From the mass-shell equation for the vector-meson momentum $K_{V}^{2}=\left(q+r_{P}\right)^{2}=M_{V}^{2}$, we find that for these reactions

$$
x_{P} \sim \frac{m_{V}^{2}+Q^{2}+|t|}{s y}
$$

and is small at high energies. This variable is not fixed for $Q \bar{Q}$ production.

We use the light-cone variables that are determined as $a_{ \pm}=a_{0} \pm a_{z}$. In these variables, the scalar production of two 4-vectors looks like

$$
a \cdot b=\frac{1}{2}\left(a_{+} b_{-}+a_{-} b_{+}\right)-\vec{a}_{\perp} \vec{b}_{\perp}
$$

where $\vec{a}_{\perp}$ and $\vec{b}_{\perp}$ represent the transverse parts of the momenta. In calculation, the center of mass system is used where the momenta of the initial lepton and proton are going along the $z$ axis and have the form

$$
l=\left(p_{+}, \frac{\mu^{2}}{p_{+}}, \overrightarrow{0}\right), \quad p=\left(\frac{m^{2}}{p_{+}}, p_{+}, \overrightarrow{0}\right) .
$$

Here $\mu$ and $m$ are the lepton and proton mass. The energy of the lepton-proton system then reads as $s \sim p_{+}^{2}$. 
The momenta are carried by the photon and the Pomeron and can be written as follows:

$$
\begin{aligned}
q & =\left(y p_{+},-\frac{Q^{2}}{p_{+}}, \vec{q}_{\perp}\right), \quad\left|q_{\perp}\right|=\sqrt{Q^{2}(1-y)} \\
r_{P} & =\left(-\frac{|t|}{p_{+}}, x_{P} p_{+}, \vec{r}_{\perp}\right), \quad\left|r_{\perp}\right|=\sqrt{|t|\left(1-x_{P}\right)} .
\end{aligned}
$$

We can determine the spin vectors with positive helicity of the lepton and the proton by

$$
\begin{aligned}
& s_{l}=\frac{1}{\mu}\left(p_{+},-\frac{\mu^{2}}{p_{+}}, \overrightarrow{0}\right), \quad s_{l}^{2}=-1, \quad s_{l} \cdot l=0 ; \\
& s_{p}=\frac{1}{m}\left(\frac{m^{2}}{p_{+}},-p_{+}, \overrightarrow{0}\right), \quad s_{p}^{2}=-1, \quad s_{p} \cdot p=0 .
\end{aligned}
$$

The polarization vector for a transversely polarized target can be written in the form

$$
s_{p}^{\perp}=\left(0,0, \vec{s}_{\perp}\right), \quad \vec{s}_{\perp}^{2}=1 .
$$

\section{Structure of Leptonic and Hadronic Tensors}

\subsection{Leptonic Tensor}

The structure of the leptonic tensor is quite simple [27], because the lepton is a point-like object

$$
\begin{aligned}
\mathcal{L}^{\mu \nu}\left(s_{l}\right) & =\sum_{\text {spin } s_{f}} \bar{u}\left(l^{\prime}, s_{f}\right) \gamma^{\mu} u\left(l, s_{l}\right) \bar{u}\left(l, s_{l}\right) \gamma^{\nu} u\left(l^{\prime}, s_{f}\right) \\
& =\operatorname{Tr}\left[(l+\mu) \frac{1+\gamma_{5} \phi_{l}}{2} \gamma^{\nu}\left(l^{\prime}+\mu\right) \gamma^{\mu}\right] .
\end{aligned}
$$

Here $l$ and $l^{\prime}$ are the initial and final lepton momenta, and $s_{l}$ is a spin vector of the initial lepton determined in (6).

The sum and difference of the cross sections with parallel and antiparallel longitudinal polarization of a proton and a lepton are expressed in terms of the spin-average and spin-dependent hadron and lepton tensors. The latter is determined by the relation

$$
\mathcal{L}^{\mu \nu}( \pm)=\frac{1}{2}\left(\mathcal{L}^{\mu \nu}\left(+\frac{1}{2}\right) \pm \mathcal{L}^{\mu \nu}\left(-\frac{1}{2}\right)\right)
$$

where $\mathcal{L}^{\mu ; \nu}\left( \pm \frac{1}{2}\right)$ are the tensors with helicity of the initial lepton equal to $\pm 1 / 2$. The tensors (1) look like

$$
\begin{aligned}
& \mathcal{L}^{\mu \nu}(+)=2\left(g^{\mu \nu} l \cdot q+2 l^{\mu} l^{\nu}-l^{\mu} q^{\nu}-l^{\nu} q^{\mu}\right), \\
& \mathcal{L}^{\mu \nu}(-)=2 i \mu \epsilon^{\mu \nu \delta \rho} q_{\delta}\left(s_{l}\right)_{\rho} .
\end{aligned}
$$



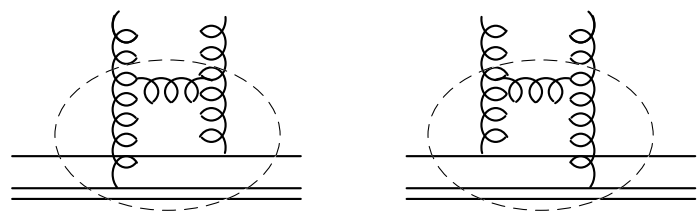

Figure 1: Graphs which give the leading Log contribution to the $g g p$ vertex in $\alpha^{2}$ order.

\subsection{Proton Two Gluon Coupling and Hadron Tensor}

The $Q \bar{Q}$ system which appears in the final state or passes into the vector meson in reaction (Ii) can be produced in two ways. The first one is the photon interaction with the $Q \bar{Q}$ state from the proton. This contribution can be connected with the quark distribution in a nucleon. The other contribution is determined by the photon-gluon fusion which produces $Q \bar{Q}$ system. The quark pair must be in a color singlet state to produce the vector meson. This means that the gluon state should be colorless too and contains two gluons at least. We are working in the low $x$ region where the gluon contribution predominates. It is associated with the Pomeron that describes diffractive processes at high energies. In QCD-inspired models, the Pomeron is usually represented as a two-gluon object.

Properties of the gluon structure functions are determined by the nonperturbative effects inside the proton. We shall analyze only the matrix structure of two-gluon coupling with the proton within the quark-diquark model [28] where the proton is composed of a quark and a diquark. Composite scalar and vector diquarks provide an effective description of nonperturbative effects in the gluon-proton interaction. The vector diguark produces spin-flip effects in the proton coupling with the gluon.

It has been shown in $29 \|$ that the leading contribution like $\alpha_{s}\left[\alpha_{s} \ln (1 / x)\right]^{n}$ to the Pomeron is determined by the gluon ladder graphs. In the $\left[\alpha_{s}\right]^{2}$ order we have in the model two ladder graphs shown in Fig. 1 with $\alpha_{s}^{2} \ln (1 / x)$ behavior. We include to the gluons coupling with the proton the gluon ladder, except two upper $t$-channel gluons in Fig. 1. This coupling is shown in the graphs of Fig. 1 by the blob. In what follows, we shall calculate the imaginary part of the Pomeron contribution to the scattering amplitude which dominates in the high-energy region. This contribution is equivalent to the $t-$ channel cut in the gluon-loop graphs. In the diquark model, the following structures in the coupling appears

$$
\begin{aligned}
V_{p g g}^{\alpha \beta}\left(p, t, x_{P}, l_{\perp}\right) & =B\left(t, x_{P}, l_{\perp}\right)\left(\gamma^{\alpha} p^{\beta}+\gamma^{\beta} p^{\alpha}\right)+\frac{i K\left(t, x_{P}, l_{\perp}\right)}{2 m}\left(p^{\alpha} \sigma^{\beta \gamma} r_{\gamma}+p^{\beta} \sigma^{\alpha \gamma} r_{\gamma}\right) \\
& +i D\left(t, x_{P}, l_{\perp}\right) \epsilon^{\alpha \beta \delta \rho} p_{\delta} \gamma_{\rho} \gamma_{5}+\ldots
\end{aligned}
$$

Here $m$ is the proton mass. In the matrix structure (11) we wrote only the terms with 
the maximal powers of a large proton momentum $p$. The structure functions in (11) are dependent on the transverse part of the gluon momentum $l_{\perp}$. The first two terms of the vertex (11) are symmetric in the gluon indices $\alpha, \beta$. The structure proportional to $B(t, \ldots)$ determines the spin-non-flip contribution. The term $\propto K(t, \ldots)$ leads to the transverse spin-flip at the vertex. The asymmetric structure in (11) is proportional to $D \gamma_{\rho} \gamma_{5}$ and can be associated with $\Delta G$. It should give a visible contribution to the double spin longitudinal asymmetry $A_{l l}$. We do not consider this structure here and concentrate on the transverse effects in the proton. In a QCD-based diquark model of the proton, the first two terms in (11) were estimated in the proton-proton scattering amplitude for moderate momentum transfer [30]. At small momentum transfer, such a model calculation is not possible, and we do not know explicitly the functions $B, K, \ldots$ in (11). Note that a coupling similar to (11) was found in high energy quark-quark scattering when large-distance effects were considered in the gluon loops [31].

In what follows, we analyse the $\gamma^{*} g g \rightarrow Q \bar{Q}$ transition amplitude. The typical momentum of quarks there is proportional to the photon momentum $q$. In the Feynman gauge, we can decompose the $g_{\mu \nu}$ tensors from $t$ - channel gluons into the longitudinal and transverse parts [29]

$$
g^{\alpha \alpha^{\prime}}=g_{l}^{\alpha \alpha^{\prime}}+g_{\perp}^{\alpha \alpha^{\prime}} \text { with } g_{l}^{\alpha \alpha^{\prime}} \sim \frac{q^{\alpha} p^{\alpha^{\prime}}}{(p q)} .
$$

The product of the $g_{l}^{\alpha \alpha^{\prime}}$ tensors by the two-gluon coupling of the proton can be written in the form

$$
g_{l}^{\alpha^{\prime} \alpha} g_{l}^{\beta^{\prime} \beta} V_{p g g}^{\alpha \beta}\left(p, t, x_{P}, l_{\perp}\right) \propto p^{\alpha^{\prime}} p^{\beta^{\prime}}\left[\frac{q}{(p q)} B\left(t, x_{P}, l_{\perp}\right)+\frac{i K\left(t, x_{P}, l_{\perp}\right)}{2 m(p q)} \sigma^{\beta \gamma} q_{\beta} r_{\gamma}\right] .
$$

The structure proportional to $D$ is asymmetric in gluon indices. It will contribute only in the case when one of the gluon tensors in (13) has a transverse component. It can be seen that the structure in square brackets in (13) is related directly to the definitions of the skewed gluon distribution (see e.g. [1]). So, one can conclude that after integration over the gluon transverse momentum $l_{\perp}$, we should have connections:

$$
\begin{aligned}
& \mathcal{F}_{\zeta}^{g}(\zeta, t) \propto \int d^{2} l_{\perp} B\left(t, \zeta=x_{P}, l_{\perp}\right) \phi\left(l_{\perp}, \ldots\right) \\
& \mathcal{K}_{\zeta}^{g}(\zeta, t) \propto \int d^{2} l_{\perp} K\left(t, \zeta=x_{P}, l_{\perp}\right) \phi\left(l_{\perp}, \ldots\right),
\end{aligned}
$$

and $B$ and $K$ are nonintegrated gluon distribution functions which describe spin-average and transverse spin effects in the proton. The universal function $\phi$ in (14) will be found later. In future calculations, we use the $g^{\alpha \alpha^{\prime}}$ tensor without its decomposition into longitudinal and transverse parts. 
The hadronic tensor is given by

$$
W^{\alpha \alpha^{\prime} ; \beta \beta^{\prime}}\left(s_{p}\right)=\sum_{\text {spin } s_{f}} \bar{u}\left(p^{\prime}, s_{f}\right) V_{p g g}^{\alpha \alpha^{\prime}}\left(p, t, x_{P}, l\right) u\left(p, s_{p}\right) \bar{u}\left(p, s_{p}\right) V_{p g g}^{\beta \beta^{\prime}+}\left(p, t, x_{P}, l^{\prime}\right) u\left(p^{\prime}, s_{f}\right)
$$

and is determined by a trace similar to (8). The spin-average and spin-dependent hadron tensors are defined as

$$
W^{\alpha \alpha^{\prime} ; \beta \beta^{\prime}}( \pm)=\frac{1}{2}\left(W^{\alpha \alpha^{\prime} ; \beta \beta^{\prime}}\left(+s_{p}\right) \pm W^{\alpha \alpha^{\prime} ; \beta \beta^{\prime}}\left(-s_{p}\right)\right) .
$$

This form is written for an arbitrary spin vector $s_{p}$ and can be used as well for transversely or longitudinally polarized target. In the last case, the contribution of $D$ structure should be considered. For the leading term of spin- average structure $W(+)$ for the ansatz (11) we find

$$
W^{\alpha \alpha^{\prime} ; \beta \beta^{\prime}}(+)=16 p^{\alpha} p^{\alpha^{\prime}} p^{\beta} p^{\beta^{\prime}}\left(|B|^{2}+\frac{|t|}{m^{2}}|K|^{2}\right) .
$$

Note that we omit, for simplicity here and in what follows, the arguments of the $B$ and $K$ functions unless it is necessary. However, we shall remember that the amplitudes $B$ and $K$ depend on $l$, otherwise the complex conjugate quantities $B^{\star}$ and $K^{\star}$ are functions of $l^{\prime}$. The obtained equation for the spin-average tensor coincides in form with the cross section of the proton off the spinless particle (a meson e.g.). Really, the meson-proton helicitynon-flip and helicity-flip amplitudes can be written in terms of the invariant functions $\tilde{B}$ and $\tilde{K}$ which describe spin-non-flip and spin-flip effects

$$
F_{++}(s, t)=i s[\tilde{B}(t)] f(t) ; \quad F_{+-}(s, t)=i s \frac{\sqrt{|t|}}{m} \tilde{K}(t) f(t) \text {, }
$$

where $f(t)$ is determined by the Pomeron coupling with meson. The functions $\tilde{B}$ and $\tilde{K}$ are defined by integrals like (14). The cross-section is written in the form

$$
\frac{d \sigma}{d t} \sim\left[|\tilde{B}(t)|^{2}+\frac{|t|}{m^{2}}|\tilde{K}(t)|^{2}\right] f(t)^{2}
$$

The term proportional to $\tilde{B}$ represents the standard Pomeron coupling that leads to the non-flip amplitude. The $\tilde{K}$ function is the spin-dependent part of the Pomeron coupling which produces in our case the spin-flip effects non vanishing at high-energies. The models [30, 32 predict a value of single spin transverse asymmetry of about $10 \%$ for $|t| \sim 3 \mathrm{GeV}^{2}$ which is of the same order of magnitude as that observed experimentally [33]. It has been found in [30, 32] that the ratio $|\tilde{K}| /|\tilde{B}| \sim 0.1$ and has a weak energy dependence. The weak energy dependence of spin asymmetries in exclusive reactions is not in contradiction with the experiment [32, 34]. 
The spin-dependent part of the hadron tensor can be written as

$$
W^{\alpha \alpha^{\prime} ; \beta \beta^{\prime}}(-)=S_{0}^{\alpha \alpha^{\prime} ; \beta \beta^{\prime}}+S_{r}^{\alpha \alpha^{\prime} ; \beta \beta^{\prime}}+A_{t}^{\alpha \alpha^{\prime} ; \beta \beta^{\prime}} .
$$

The functions $S$ are symmetric in $\alpha, \alpha^{\prime}$ and $\beta, \beta^{\prime}$ indices

$$
S_{0}^{\alpha \alpha^{\prime} ; \beta \beta^{\prime}}=8 i \frac{B K^{*}-B^{*} K}{m} p^{\beta} p^{\beta^{\prime}} \Gamma^{\alpha \alpha^{\prime}}
$$

and

$$
\begin{aligned}
S_{r}^{\alpha \alpha^{\prime} ; \beta \beta^{\prime}} & =2 i \frac{B^{*} K}{m}\left(p^{\alpha}\left(r_{P}\right)^{\alpha^{\prime}}+p^{\alpha^{\prime}}\left(r_{P}\right)^{\alpha}\right) \Gamma^{\beta \beta^{\prime}} \\
& -2 i \frac{B K^{*}}{m}\left(p^{\beta}\left(r_{P}\right)^{\beta^{\prime}}+p^{\beta^{\prime}}\left(r_{P}\right)^{\beta}\right) \Gamma^{\alpha \alpha^{\prime}}
\end{aligned}
$$

Here

$$
\Gamma^{\alpha \alpha^{\prime}}=p^{\alpha} \epsilon^{\alpha^{\prime} \gamma \delta \rho} p_{\gamma}\left(r_{P}\right)_{\delta}\left(s_{p}\right)_{\rho}+p^{\alpha^{\prime}} \epsilon^{\alpha \gamma \delta \rho} p_{\gamma}\left(r_{P}\right)_{\delta}\left(s_{p}\right)_{\rho}
$$

The function $A_{t}$ is asymmetric in indices

$$
\begin{aligned}
A_{t}^{\alpha \alpha^{\prime} ; \beta \beta^{\prime}} & =2 i|t| \frac{B^{*} K}{m}\left[p^{\alpha} p^{\beta} \epsilon^{\alpha^{\prime} \beta^{\prime} \delta \rho} p_{\delta}\left(s_{p}\right)_{\rho}+p^{\alpha} p^{\beta^{\prime}} \epsilon^{\alpha^{\prime} \beta \delta \rho} p_{\delta}\left(s_{p}\right)_{\rho}\right. \\
& \left.+p^{\alpha^{\prime}} p^{\beta} \epsilon^{\alpha \beta^{\prime} \delta \rho} p_{\delta}\left(s_{p}\right)_{\rho}+p^{\alpha^{\prime}} p^{\beta^{\prime}} \epsilon^{\alpha \beta \delta \rho} p_{\delta}\left(s_{p}\right)_{\rho}\right]
\end{aligned}
$$

Note that these forms are general and can be used for different polarization vectors of the proton. For longitudinal proton polarization, the structure $D$ should be considered in addition.

\section{Diffractive Vector Meson Leptoproduction}

Now we proceed to analyze of the amplitude of vector meson production through the photon-two gluon fusion. In what follows, we will regard mainly the $J / \Psi$ meson production. This meson can be considered as an $S$-wave system of heavy $c \bar{c}$ quarks [35]. The $J / \Psi$-wave function in this case has the form

$$
\Psi_{V}=g\left(k+m_{q}\right) \gamma_{\mu}
$$

where $k$ is the momentum of a quark, and $m_{q}$ is its mass. In the nonrelativistic approximation, both the quarks have the same momenta $k$ equal to half of the vector meson momentum $K_{J}$, and the mass of $c$ quark is equal to $m_{J} / 2$. The transverse quark motion is not considered. This means that the vector meson distribution amplitude is approximated by the simple form $\delta(\tau-1 / 2) \delta\left(k_{t}^{2}\right)$. The constant $g$ in the wave function can be expressed through the $e^{+} e^{-}$decay width of the $J / \Psi$ meson [10

$$
g^{2}=\frac{3 \Gamma_{e^{+} e^{-}}^{J} m_{J}}{64 \pi \alpha^{2}} .
$$




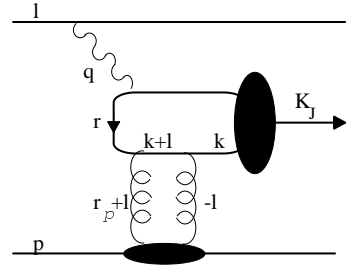

(a)

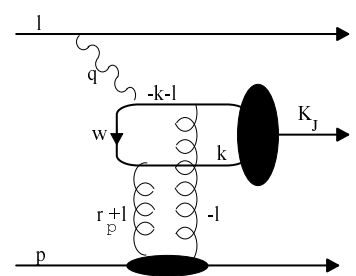

(b)

Figure 2: Two-gluon contribution to diffractive vector meson production..

The leading twist wave function (25) produces both amplitudes with longitudinal and transverse vector meson polarization because of nonzero mass $m_{q}$. For the light meson production $m_{q}=0$, and one must consider the higher twist effects to calculate the amplitude with transverse vector meson polarization (see e.g. [18).

It is known (see [10, 11] e.g.) that the leading terms of the amplitude of diffractive vector meson production is mainly imaginary. We shall consider here only the imaginary parts of the amplitudes. In this case, only the graphs of Fig.2 contribute. The gluons are coupled with single and different quarks in the $c \bar{c}$ loop (see Fig. 2 a, b). To calculate the imaginary part of the amplitude, we should consider the $\delta$-function contribution in the $s$-channel propagators $\left(k+l\right.$ and $p^{\prime}-l$ lines for Fig $⿴$ a). With the help of $\delta$ functions the integration over $l$

$$
\int d^{4} l=\frac{1}{2} \int d l_{+} d l_{-} d l_{\perp}
$$

can be carried out over $l_{+}$and $l_{-}$variables. One can find that both the $l_{ \pm}$components of the vector $l$ are small: $l_{+} \sim l_{-} \propto 1 / p_{+}$. This results in the transverse character of the gluon momentum $l^{2} \simeq-l_{\perp}^{2}$. The same is true for integration over $l$ in the nonplanar graph of Fig 2. b. For the arguments in the off mass shell quark propagators of Fig 2. a, b, we find

$$
\begin{aligned}
r^{2}-m_{q}^{2} & =-\frac{M_{J}^{2}+Q^{2}+|t|}{2} \\
w^{2}-m_{q}^{2} & =-2\left(l_{\perp}^{2}+\vec{l}_{\perp} \vec{r}_{\perp}+\frac{M_{J}^{2}+Q^{2}+|t|}{4}\right) .
\end{aligned}
$$

Thus, these quark lines are far from the mass shell for heavy vector meson production even for small $Q^{2}[10]$.

In what follows we calculate the polarized cross section of vector meson production. The cross section can be represented as a square of the $\gamma^{\star} g g \rightarrow V$ amplitude convoluted with the lepton and hadron polarized tensors. Some details of calculations conducted for longitudinal target polarization can be found in [36]. 
We consider both longitudinal and transverse polarization of the vector meson which can be carried out directly for $J / \Psi$ production for the wave function (25). For the sum over polarization of $J / \Psi$ polarized vectors $e_{J}$ we have

$$
\sum_{\operatorname{Spin}_{J}} e_{J}^{\rho}\left(e_{J}^{\sigma}\right)^{+}=-g^{\rho \sigma}+\frac{K_{J}^{\rho} K_{J}^{\sigma}}{m_{J}^{2}}
$$

The spin-average and spin-dependent cross-sections of vector meson leptoproduction with longitudinal polarization of a lepton and transverse polarization of the proton are determined by the relation

$$
d \sigma( \pm)=\frac{1}{2}(d \sigma(\rightarrow \Downarrow) \pm d \sigma(\rightarrow \Uparrow))
$$

The cross section $d \sigma( \pm)$ can be written in the form

$$
\frac{d \sigma^{ \pm}}{d Q^{2} d y d t}=\frac{\left|T^{ \pm}\right|^{2}}{32(2 \pi)^{3} Q^{4} s^{2} y} .
$$

For the spin-average amplitude squared we find

$$
\left|T^{+}\right|^{2}=\frac{s^{2} N}{4 \bar{Q}^{4}}\left(\left(1+(1-y)^{2}\right) m_{V}^{2}+2(1-y) Q^{2}\right)\left[|\tilde{B}|^{2}+|\tilde{K}|^{2} \frac{|t|}{m^{2}}\right] .
$$

Here $\bar{Q}^{2}=\left(m_{V}^{2}+Q^{2}+|t|\right) / 4$, and $N$ is the normalization factor

$$
N=\frac{\Gamma_{e^{+} e^{-}}^{J} M_{J} \alpha_{s}^{4}}{27 \pi^{2}}
$$

The term proportional to $\left(1+(1-y)^{2}\right) m_{V}^{2}$ in $(32)$ represents the contribution of the virtual photon with transverse polarization. The $2(1-y) Q^{2}$ term describes the effect of longitudinal photons. This contribution is predominant for high $Q^{2}$. The $\tilde{B}$ and $\tilde{K}$ functions are expressed through the integral over the transverse momentum of the gluon. The function $\tilde{B}$ is determined by

$$
\begin{aligned}
\tilde{B} & =\bar{Q}^{2} \int \frac{d^{2} l_{\perp}\left(l_{\perp}^{2}+\vec{l}_{\perp} \vec{r}_{\perp}\right) B\left(t, l_{\perp}^{2}, x_{P}, \ldots\right)}{\left(l_{\perp}^{2}+\lambda^{2}\right)\left(\left(\vec{l}_{\perp}+\vec{\Delta}\right)^{2}+\lambda^{2}\right)\left[l_{\perp}^{2}+\vec{l}_{\perp} \vec{r}_{\perp}+\bar{Q}^{2}\right]} \\
& \sim \int_{0}^{l_{\perp}^{2}<\bar{Q}^{2}} \frac{d^{2} l_{\perp}\left(l_{\perp}^{2}+\vec{l}_{\perp} \vec{r}_{\perp}\right)}{\left(l_{\perp}^{2}+\lambda^{2}\right)\left(\left(\vec{l}_{\perp}+\vec{r}_{\perp}\right)^{2}+\lambda^{2}\right)} B\left(t, l_{\perp}^{2}, x_{P}, \ldots\right),
\end{aligned}
$$

We find that cross sections depend on the variable $\bar{Q}^{2}$ which is a modification of the scale variable proposed in [10, 11] for the case of large momentum transfer. The term $\left(l_{\perp}^{2}+\vec{l}_{\perp} \vec{r}_{\perp}\right)$ appears in the numerator of (34) because of the cancellation between the planar and nonplanar graphs where gluons are coupled with single and different quarks 
(Fig.2). The $\tilde{K}$ function is determined by a similar integral. The integral (34) can be connected with the gluon SPD as

$$
\mathcal{F}_{x_{P}}^{g}\left(x_{P}, t, \bar{Q}^{2}\right) \sim \int_{0}^{l_{\perp}^{2}<\bar{Q}^{2}} \frac{d^{2} l_{\perp}\left(l_{\perp}^{2}+\vec{l}_{\perp} \vec{r}_{\perp}\right)}{\left(l_{\perp}^{2}+\lambda^{2}\right)\left(\left(\vec{l}_{\perp}+\vec{r}_{\perp}\right)^{2}+\lambda^{2}\right)} B\left(t, l_{\perp}^{2}, x_{P}, \ldots\right)=\tilde{B} .
$$

Note that if one considers effects of the transverse quark motion in the the vector meson wave function, the scale variable in SPD will be changed to $\bar{Q}^{2} \rightarrow \bar{Q}^{2}+k_{\perp}^{2}$ [13, 37. Thus, the $B\left(l_{\perp}^{2}, x_{P}, \ldots\right)$ function is a nonintegrated spin- average gluon distribution. The $\tilde{K}$ function is proportional to the $\mathcal{K}_{x_{P}}^{g}\left(x_{P}, t\right)$ distribution. The function $\phi$ in (14) has the form

$$
\phi\left(l_{\perp}, \ldots\right)=\frac{\left(l_{\perp}^{2}+\vec{l}_{\perp} \vec{r}_{\perp}\right)}{\left(l_{\perp}^{2}+\lambda^{2}\right)\left(\left(\vec{l}_{\perp}+\vec{r}_{\perp}\right)^{2}+\lambda^{2}\right)} .
$$

The spin-dependent amplitude squared looks like

$$
\left|T^{-}\right|^{2}=\frac{\vec{Q} \vec{S}_{\perp}}{4 m} \frac{s|t| N}{4 \bar{Q}^{4}}\left(Q^{2}+m_{V}^{2}+|t|\right) \frac{\tilde{B} \tilde{K}^{*}+\tilde{B}^{*} \tilde{K}}{2} .
$$

We shall use the spin-dependent cross sections obtained here for the numerical analysis of polarized vector meson production in section 6 .

\section{Diffractive $Q \bar{Q}$ Photoproduction}

Let us study now the diffractive $Q \bar{Q}$ production in the lepton-proton reaction. This process is determined by similar graphs shown in Fig. 2. The change is in the photontwo-gluon fusion amplitude where we do not project the $Q \bar{Q}$ state onto the vector meson. The quark-antiquark contribution, instead of $t$ - channel gluons, is possible for light quark production. To suppress this contribution of the quark structure function which should be essential at large $x$, we investigate quark production at small $x \leq 0.1$. In this kinematical region the gluon contribution is predominant.
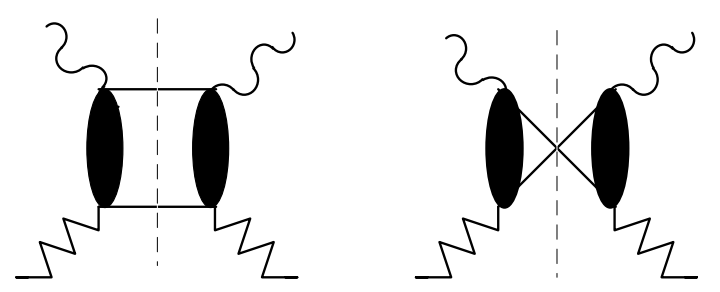

Figure 3: Box graphs contributions to the cross section of diffractive $Q \bar{Q}$ production.

As in the case of vector meson production, we calculate the spin average and spindependent cross section (31) of diffractive $Q \bar{Q}$ leptoproduction. To calculate these cross 
sections, we should integrate the corresponding amplitudes squared over the $Q \bar{Q}$ phase space $d N_{Q \bar{Q}}=\Pi_{f} \frac{d^{3} p_{f}}{(2 \pi)^{3} 2 E_{f}}$ with the delta function that reflects the momentum conservation. It can be easily seen that

$$
\frac{d^{3} p_{1}}{2 E_{1}} \frac{d^{3} p_{2}}{2 E_{2}} \delta^{4}\left(q+r_{P}-p_{1}-p_{2}\right)=d^{4} p_{1} \delta\left(p_{1}^{2}-m_{q}^{2}\right) \delta\left(p_{2}^{2}-m_{q}^{2}\right)
$$

and the calculation of $\gamma g g \rightarrow Q \bar{Q}$ cross section is equivalent to computation of the imaginary part of the quark loop diagram shown in Fig. 3. The amplitude of photon-two-gluon fusion shown by blobs in Fig. 3 represents a sum of graphs in Fig. 4. The diagrams of Fig. are similar to the planar and nonplanar gluon graphs of Fig.2. As a result, the gluon contribution to the cross section should be similar to that obtained in (34).

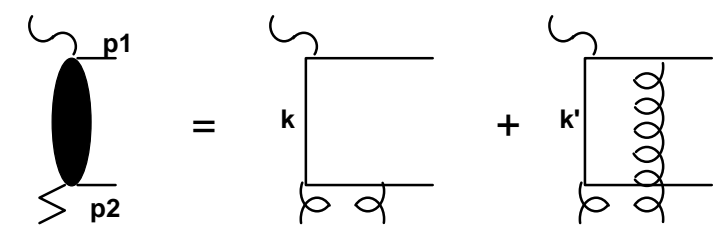

Figure 4: The amplitude of photon-two-gluon fusion

The final quark momenta $p_{1}, p_{2}$ and the momentum of the off-mass-shell quark $k\left(k^{\prime}\right)$ can be determined with the help of delta functions in (38). There are two integration regions for $k$ vectors. For Region I, we find that $p_{1} \sim q$ and $p_{2} \sim r_{P}$

$$
\begin{aligned}
& p_{1} \sim\left(y p_{+}-\frac{|t|}{p_{+}}-\frac{m_{q}^{2}+\left(\vec{r}_{\perp}+\vec{k}_{\perp}\right)^{2}}{p_{+} x_{P}}, \frac{m_{q}^{2}+\left(\vec{q}_{\perp}-\vec{k}_{\perp}\right)^{2}}{p_{+} y},\left(\vec{q}_{\perp}-\vec{k}_{\perp}\right)\right), \\
& p_{2} \sim\left(\frac{m_{q}^{2}+\left(\vec{r}_{\perp}+\vec{k}_{\perp}\right)^{2}}{p_{+} x_{P}}, x_{P} p_{+}-\frac{Q^{2}}{p_{+}}-\frac{m_{q}^{2}+\left(\vec{q}_{\perp}-\vec{k}_{\perp}\right)^{2}}{p_{+} y},\left(\vec{r}_{\perp}+\vec{k}_{\perp}\right)\right)
\end{aligned}
$$

and vector $k$ is mainly transverse: $k^{2} \sim-k_{\perp}^{2}$. The $t$ channel gluon contribution is predominated if both quark-proton energies are large. We find that

$$
\left(p_{1}+p\right)^{2} \sim y p_{+}^{2} \quad\left(p_{2}+p\right)^{2} \sim \frac{m_{q}^{2}+k_{\perp}^{2}}{x_{P}} .
$$

Thus, $x_{P}$ should be quite small $\left(x_{P} \leq 0.1\right)$. At the same, time $k_{\perp}^{2}$ should be not small (we shall use $k_{\perp}^{2}>1 \mathrm{GeV}^{2}$ ). A non small value for $k^{2}$ produces the large quark virtuality for the graphs of Fig. (田).

For Region II, the quarks momentum changed places $p_{1} \leftrightarrow p_{2}$. In this case, the vector $k$ has a large longitudinal component, and $k^{2} \sim-x_{P} p_{+}^{2}$. One can suppose that such contributions should be suppressed. However, in this case, we find a similar large variable 
from trace in the numerator of the diagram that compensates $p_{+}^{2}$ in denominator. A similar compensation between the numerator and denominator takes place for the nonplanar quark loop diagrams (the second graph in Fig.3). In this case, we have a large variable only in one propagator. The calculation of $\gamma g g \rightarrow Q \bar{Q}$ process is more complicated than the vector meson case. We must consider here 8 graphs with two Regions for quark momenta. This generates a complete set of graphs of $Q \bar{Q}$ production.

The integration over quark momenta $k_{ \pm}$in the loop can be carried out with the help of delta functions in (38)

$$
d^{4} k \delta\left(p_{1}^{2}-m_{q}^{2}\right) \delta\left(p_{2}^{2}-m_{q}^{2}\right) \sim \frac{d^{2} k_{\perp}}{M_{X}^{2} \sqrt{1-4\left(k_{\perp}^{2}+m_{q}^{2}\right) / M_{X}^{2}}}
$$

As a result, the spin-average and spin-dependent cross section can be written in the form

$$
\frac{d^{5} \sigma( \pm)}{d Q^{2} d y d x_{p} d t d k_{\perp}^{2}}=\left(\begin{array}{c}
\left(2-2 y+y^{2}\right) \\
(2-y)
\end{array}\right) \frac{C\left(x_{P}, Q^{2}\right) N( \pm)}{\sqrt{1-4\left(k_{\perp}^{2}+m_{q}^{2}\right) / M_{X}^{2}}} .
$$

Here $C\left(x_{P}, Q^{2}\right)$ is a normalization function which is common for the spin average and spin dependent cross section; $N( \pm)$ is determined by a sum of graphs in Fig. 3, ๑ integrated over the gluon momenta $l$ and $l^{\prime}$

$$
N( \pm)=\int \frac{d^{2} l_{\perp} d^{2} l_{\perp}^{\prime}\left(l_{\perp}^{2}+\vec{l}_{\perp} \vec{r}_{\perp}\right)\left(\left(l_{\perp}^{\prime}\right)^{2}+\vec{l}_{\perp}^{\prime} \vec{r}_{\perp}\right) D^{ \pm}\left(t, Q^{2}, l_{\perp}, l_{\perp}^{\prime}, \cdots\right)}{\left(l_{\perp}^{2}+\lambda^{2}\right)\left(\left(\vec{l}_{\perp}+\vec{r}_{\perp}\right)^{2}+\lambda^{2}\right)\left(l_{\perp}^{\prime 2}+\lambda^{2}\right)\left(\left(\vec{l}_{\perp}^{\prime}+\vec{r}_{\perp}\right)^{2}+\lambda^{2}\right)} .
$$

The $D^{ \pm}$function here is a sum of traces over the quark loops of the graphs in Figs. 3 , 4 convoluted with the spin average and spin-dependent tensors. The calculation shows a considerable cancellation between the planar and nonplanar contribution of the graphs in Fig. As a result, in the numerator of (43) we find the terms proportional to the gluon momenta $l_{\perp}$ and $l_{\perp}^{\prime}$ as in the case of vector meson production (34).

For simplicity we write the analytic forms of the graph contribution to the cross sections in the limit $\beta \rightarrow 0$. The numerical calculation will be fulfiled for arbitrary $\beta$. The contribution of the sum of the graphs of Figs. 3, 4 to the $D^{+}$function for Region I can be written in the form

$$
D_{I}^{+}=\frac{Q^{2}\left(|B|^{2}+|t| / m^{2}|K|^{2}\right)\left(\left(k_{\perp}+r_{\perp}\right)^{2}+m_{q}^{2}\right)}{\left(k_{\perp}^{2}+m_{q}^{2}\right)\left(\left(k_{\perp}-l_{\perp}\right)^{2}+m_{q}^{2}\right)\left(\left(k_{\perp}-l_{\perp}^{\prime}\right)^{2}+m_{q}^{2}\right)} .
$$

This function contains a product of the off-mass-spell quark propagators in the graphs Fig. 3, 4. We see that the quark virtuality here is quite different as compared to the vector meson case. We have no the terms proportional to $Q^{2}$ as in (28). 
This will change the scale in corresponding gluon structure functions. Really, denominators in (44) determine the effective integration region over $l$ and $l^{\prime}$ in (33). We can rewrite approximately the contribution of $D^{p}(+)$ to $N(+)$

$$
N^{p}(+) \sim \frac{\left(|\tilde{B}|^{2}+|t| / m^{2}|\tilde{K}|^{2}\right)\left(\left(k_{\perp}+r_{\perp}\right)^{2}+m_{q}^{2}\right)}{\left(k_{\perp}^{2}+m_{q}^{2}\right)^{3}}
$$

with

$$
\tilde{B} \sim \int_{0}^{l_{\perp}^{2}<k_{0}^{2}} \frac{d^{2} l_{\perp}\left(l_{\perp}^{2}+\vec{l}_{\perp} \vec{r}_{\perp}\right)}{\left(l_{\perp}^{2}+\lambda^{2}\right)\left(\left(\vec{l}_{\perp}+\vec{r}_{\perp}\right)^{2}+\lambda^{2}\right)} B\left(t, l_{\perp}^{2}, x_{P}, \ldots\right)=\mathcal{F}_{x_{P}}^{g}\left(x_{P}, t, k_{0}^{2}\right)
$$

and

$$
\tilde{K} \sim \int_{0}^{l_{\perp}^{2}<k_{0}^{2}} \frac{d^{2} l_{\perp}\left(l_{\perp}^{2}+\vec{l}_{\perp} \vec{r}_{\perp}\right)}{\left(l_{\perp}^{2}+\lambda^{2}\right)\left(\left(\vec{l}_{\perp}+\vec{r}_{\perp}\right)^{2}+\lambda^{2}\right)} K\left(t, l_{\perp}^{2}, x_{P}, \ldots\right)=\mathcal{K}_{x_{P}}^{g}\left(x_{P}, t, k_{0}^{2}\right)
$$

with $k_{0}^{2} \sim k_{\perp}^{2}+m_{q}^{2}$. For nonzero $\beta$ this scale is changed to $k_{0}^{2} \sim \frac{k_{\perp}^{2}+m_{q}^{2}}{1-\beta}$ and coincides with that found in [21, 22]. As we have expected, the gluon structure functions are determined by the camo intorrale ac in (5) hut nn a difforant crals

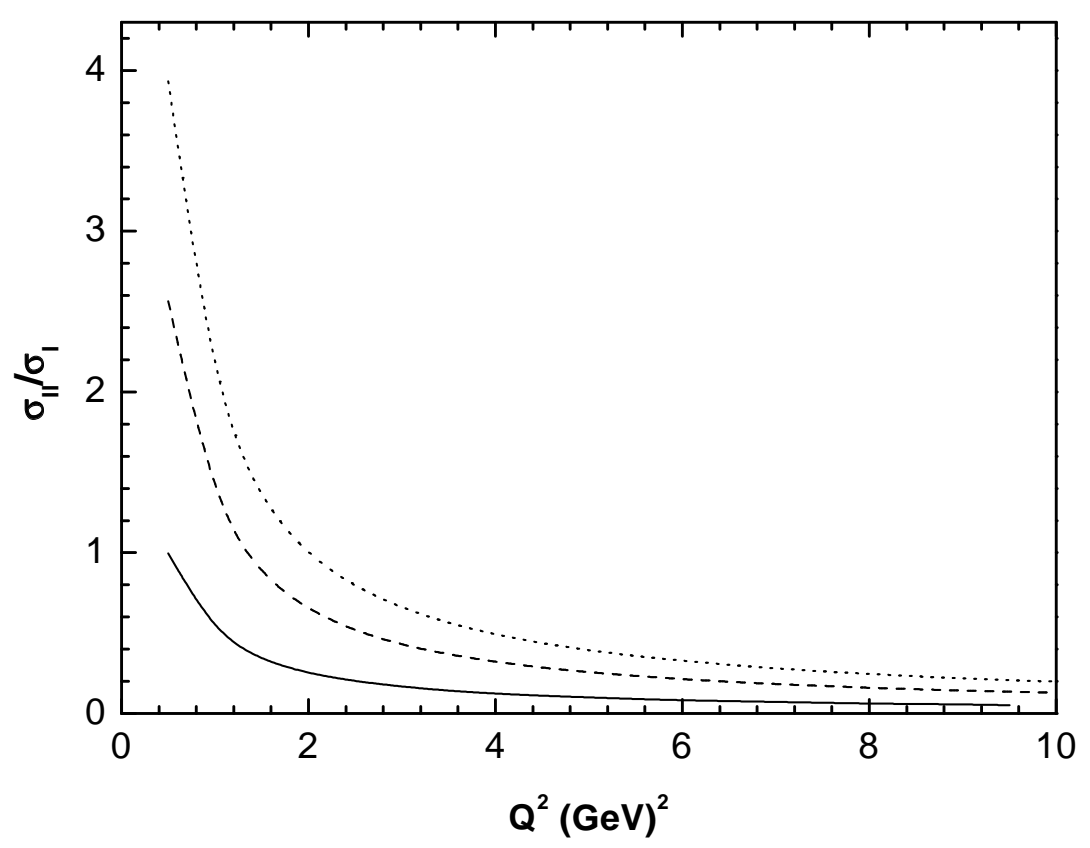

Figure 5: The ratio of cross sections for Regions II and I at $\sqrt{s}=20 \mathrm{GeV}$ for $x_{P}=0.1$, $y=0.5,|t|=0.3 \mathrm{GeV}^{2}$ : solid line- for $k^{2}=1 \mathrm{GeV}^{2}$, dashed line- for $k^{2}=3 \mathrm{GeV}^{2}$, dotted line- for $k^{2}=5 \mathrm{GeV}^{2}$.

For Region II, only the first planar graph of Fig. 3 contributes. As a result of compensation of the gluon contributions shown in Fig. 困, the form (43) is valid here too. The graphs here have lines with large quark virtuality. Propagators of these lines become 
pointlike. As a result, the contribution to the cross section for Region II has different $Q^{2}$ and $k^{2}$ dependence with respect to Region I. We find

$$
D_{I I}^{+}=\frac{2(1-y)\left(|B|^{2}+|t| / m^{2}|K|^{2}\right)}{\left(2-2 y+y^{2}\right)\left(\left(k_{\perp}+r_{\perp}\right)^{2}+m_{q}^{2}\right)} \text {. }
$$

The ratio of the cross sections for Regions I and II is shown in Fig 5. The ratio is growing with $k^{2}$. We find that the integration region II is essential at small $Q^{2}$. It can be seen that the contribution of the $D$ functions (44,48) to the cross section (42) is proportional to $D_{I}^{+} \propto\left(2-2 y+y^{2}\right), D_{I I}^{+} \propto 2(1-y)$. They represent, as in (32), the contributions with transverse and longitudinal photon polarization, respectively [21].

The contribution of all graphs to the function $N(+)$ can be written as

$$
N(+)=\left(|\tilde{B}|^{2}+|t| / m^{2}|\tilde{K}|^{2}\right) \Pi^{(+)}\left(t, k_{\perp}^{2}, Q^{2}\right)
$$

The function $\Pi^{(+)}$for nonzero $\beta$ is complicated in form and will be calculated numerically.

The same procedure is used in calculations of the spin-dependent cross sections. The spin-dependent leptonic and hadronic tensors (10,20) are used in this case. The spindependent part of the hadronic tensor (20) is much more complicated as compared with (17). This results in tangled analytic expressions of the spin-dependent cross sections. We shall discuss here only the general structure of this observable. In addition to the term observed in (37) and proportional to the scalar production $\vec{Q} \vec{S}_{\perp}$, the new term $\propto \vec{k}_{\perp} \vec{S}_{\perp}$ appears. As a result, we find the following representation of the function $N(-)$

$$
N(-)=\sqrt{\frac{|t|}{m^{2}}}\left(\tilde{B} \tilde{K}^{*}+\tilde{B}^{*} \tilde{K}\right)\left[\frac{\left(\vec{Q} \vec{S}_{\perp}\right)}{m} \Pi_{Q}^{(-)}\left(t, k_{\perp}^{2}, Q^{2}\right)+\frac{\left(\vec{k}_{\perp} \vec{S}_{\perp}\right)}{m} \Pi_{k}^{(-)}\left(t, k_{\perp}^{2}, Q^{2}\right)\right] .
$$

The second term in (50) cannot be found in the vector meson production, because we

should integrate there the amplitudes over $d^{2} k_{\perp}$. The functions $\Pi_{Q(k)}^{(-)}$will be calculated numerically as the function $\Pi^{(+)}$.

\section{Numerical Results for Vector Meson Leptoproduc- tion}

We shall calculate the polarized cross section (30) of diffractive $J / \Psi$ production determined by the amplitudes (32, 37). The spin-average cross section of the vector meson production at a small momentum transfer is proportional to the $|\tilde{B}|^{2}$ function (32) which is connected with the skewed gluon distribution (35). This result is in accordance with 
the imaginary part of the amplitude found on the basis of the SPD approach [1]. We use here a simple parameterization of the SPD as a product of the form factor and the ordinary gluon distribution

$$
\tilde{B}\left(t, x_{P}, \bar{Q}^{2}\right)=F_{B}(t)\left(x_{P} G\left(x_{P}, \bar{Q}^{2}\right)\right) .
$$

where for simplicity the form factor $F_{B}(t)$ is chosen as the electromagnetic form factor of the proton. Such a simple choice can be justified by that the Pomeron-proton vertex might be similar to the photon-proton coupling [38, 39]

$$
F_{B}(t) \sim F_{p}^{e m}(t)=\frac{\left(4 m_{p}^{2}+2.8|t|\right)}{\left(4 m_{p}^{2}+|t|\right)\left(1+|t| / 0.7 G e V^{2}\right)^{2}} .
$$

To perform simple estimations, we shall use our results from [30, 32] where it was found that the ratio of spin asymmetries in exclusive reactions at a small momentum transfer may have a weak energy dependence. The corresponding asymmetries are proportional to the ratio $|\tilde{K}| /|\tilde{B}|$ at small $x \sim 1 / s$. We shall suppose that this is true for the ratio of spin-dependent and spin-average densities in our case too and

$$
\frac{|\tilde{K}|}{|\tilde{B}|} \sim 0.1
$$

We shall use this value in our estimation of spin asymmetries of hadron leptoproduction at small $x$.

The energy dependence of the cross sections is determined by the Pomeron contribution to the gluon distribution function at small $x$

$$
\left(x_{P} G\left(x_{P}, \bar{Q}^{2}\right)\right) \sim \frac{\text { const }}{x_{P}^{1-\alpha_{p}(t)}} \sim\left(\frac{s y}{m_{J}^{2}+Q^{2}+|t|}\right)^{\left(\alpha_{p}(t)-1\right)} .
$$

Here $\alpha_{p}(t)$ is a Pomeron trajectory which is chosen in the form

$$
\alpha_{p}(t)=1+\epsilon+\alpha^{\prime} t
$$

with $\epsilon=0.15$ and $\alpha^{\prime}=0$. These values are in accordance with the fit of diffractive $J / \Psi$ production by ZEUS 40]

The typical scale of the reaction is determined by $\bar{Q}^{2}=\left(m_{J}^{2}+Q^{2}+|t|\right) / 4$. For not large $Q^{2}$ and $|t|$, the value of $\bar{Q}^{2}$ is about 2.5-3.0 $\mathrm{GeV}^{2}$. In this region, we can work with fixed $\alpha_{s} \sim 0.3$. An effective gluon mass in (34) is chosen to be equal to $0.3 \mathrm{GeV}^{2}$. The cross section depends on this parameter weakly. The value of $\Gamma_{e^{+} e^{-}}^{J}=5.26 \mathrm{keV}$ is used. The predicted cross sections are shown in Fig. 6. Our results reproduce experimental data quite well. 


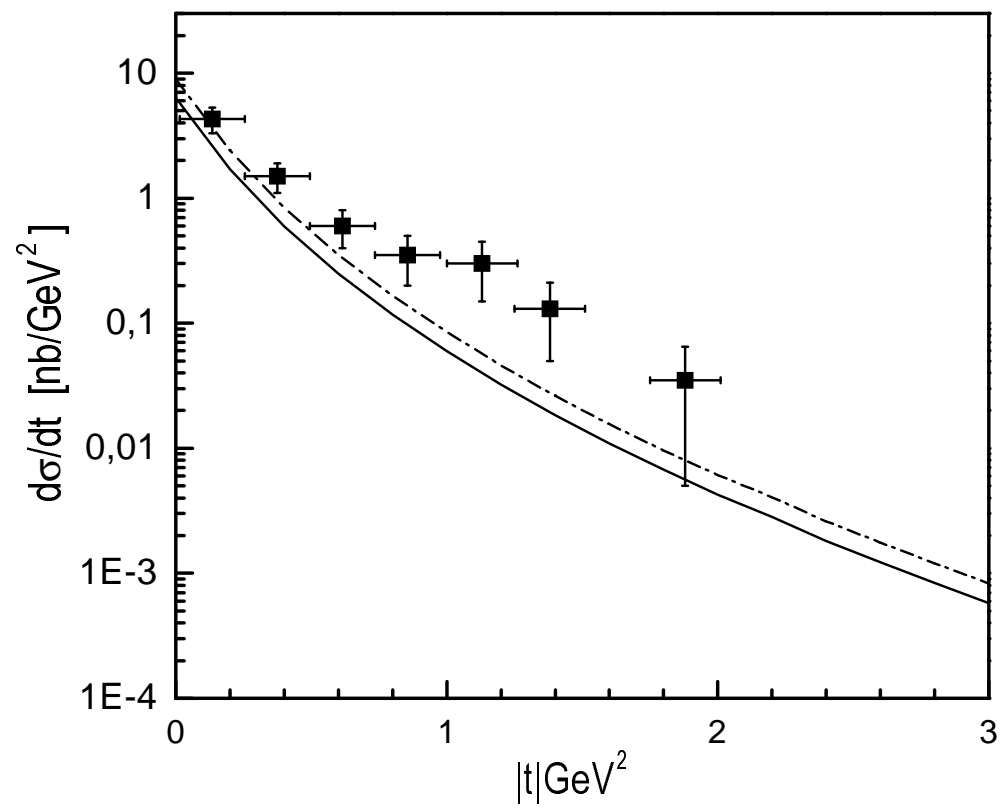

Figure 6: The differential cross section of $J / \Psi$ production at HERA energy: solid line -for $|\tilde{K}| /|\tilde{B}|=0$; dot-dashed line -for $|\tilde{K}| /|\tilde{B}|=0.1$. Data are from $[6]$.

The $A_{l T}$ asymmetry for vector meson production is determined by the ratio of cross sections determined in (37,32)

$$
A_{l T}=\frac{\sigma(-)}{\sigma(+)} \sim \frac{\vec{Q} \vec{S}_{\perp}}{4 m} \frac{y x_{P}|t|}{\left(1+(1-y)^{2}\right) m_{V}^{2}+2(1-y) Q^{2}} \frac{\tilde{B} \tilde{K}}{|\tilde{B}|^{2}+|\tilde{K}|^{2}|t| / m^{2}} .
$$

For a small momentum transfer, this asymmetry can be approximated as

$$
A_{L T} \sim C_{g} \frac{\mathcal{K}_{\zeta}^{g}(\zeta)}{\mathcal{F}_{\zeta}^{g}(\zeta)} \quad \text { with } \zeta=x_{P}
$$

Simple estimations show that the coefficient $C_{g}(J / \Psi)$ at HERMES energy for $y=0.5,|t|=$ $1 \mathrm{GeV}^{2}, Q^{2}=5 \mathrm{GeV}^{2}$ is quite small, about 0.007 . To get expected values for coefficients (57) for light vector mesons, we use the same Eq. (56). The simple model used for the wave function predicts weak mass dependence of the gluon contribution to the asymmetry. For the same kinematical variables, $C(\phi) \sim C(\rho) \sim 0.008$. However, these results are obtained for the nonrelativistic meson wave function of the form $\delta(\tau-1 / 2) \delta\left(k_{t}^{2}\right)$ that is not a good approximation for light meson production. To get suitable predictions for $\rho, \phi$ meson production, it is important to study a more realistic wave function and take into consideration the transverse quark degrees of freedom. Moreover, for $\rho, \phi$ meson production, the contribution of the quark SPD should be considered in the HERMES energy range. 


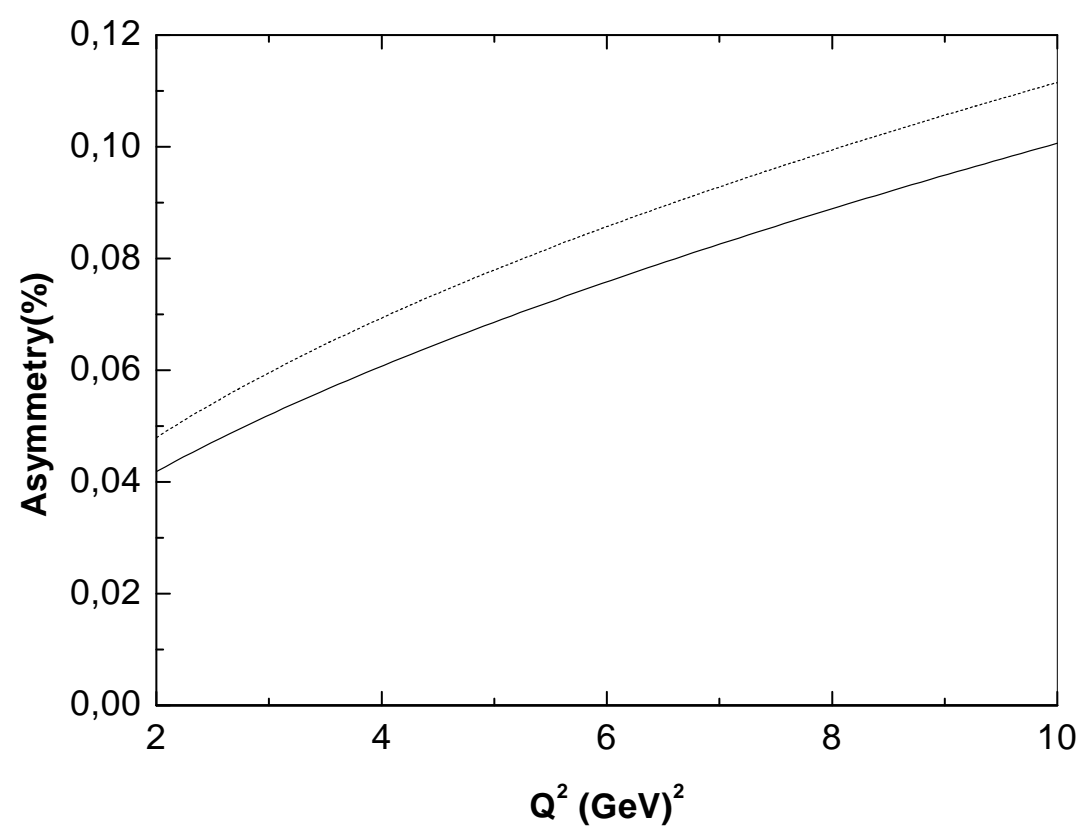

Figure 7: The $A_{l T}$ asymmetry for vector meson production at $\sqrt{s}=7 \mathrm{GeV}(y=0.5,|t|=$ $\left.1 \mathrm{GeV}^{2}\right)$ : solid line -for $J / \Psi$ production; dotted line -for $\rho$ production.

The asymmetry predicted for $J / \Psi$ production at HERMES energies is shown in Fig. 7 $(\tilde{K} / \tilde{B}=0.1)$ for the case when the transverse part of the photon momentum is parallel to the target polarization $S_{\perp}$. Simple estimations on the basis of (57) for $\rho$ meson production are shown there too. At the HERA energies, asymmetry will be extremely small.

\section{Predictions for $Q \bar{Q}$ Leptoproduction}

We shall discuss here our prediction for polarized diffractive $Q \bar{Q}$ production. We do not consider the cross section but only the asymmetry $A_{l T}=\sigma(-) / \sigma(+)$. In estimations we shall use the same parameterizations of SPD as in (51) with the functions determined in (52). As in the case of vector meson production, the asymmetry is approximately proportional to the ratio of polarized and spin-average gluon distribution functions

$$
A_{L T}^{Q \bar{Q}} \sim C^{Q \bar{Q}} \frac{\mathcal{K}_{\zeta}^{g}(\zeta)}{\mathcal{F}_{\zeta}^{g}(\zeta)} \text { with } \zeta=x_{P}
$$

As previously, in our estimations we use the value $|\tilde{K}| /|\tilde{B}| \sim 0.1$.

The spin-dependent contribution has two terms proportional to the scalar products $\vec{k}_{\perp} \vec{S}_{\perp}$ and $\vec{Q} \vec{S}_{\perp}$ (50). We shall study these contributions to asymmetry separately. The first term will be analyzed for the case when the transverse jet momentum $\vec{k}_{\perp}$ is parallel to the target polarization $\vec{S}_{\perp}$. The asymmetry is maximal in this case. We would like 


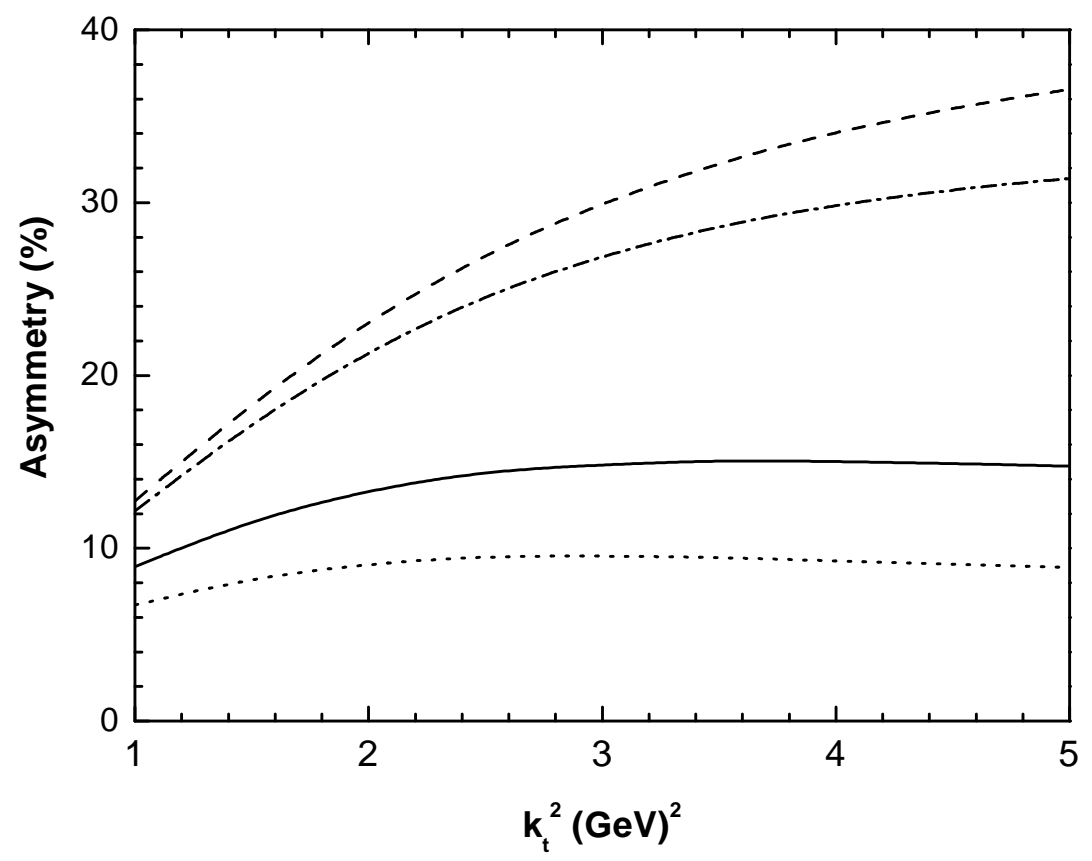

Figure 8: The $A_{l T}^{k}$ asymmetry in diffractive light $Q \bar{Q}$ production at $\sqrt{s}=20 \mathrm{GeV}$ for $x_{P}=$ $0.1, y=0.5,|t|=0.3 \mathrm{GeV}^{2}$ : dotted line-for $Q^{2}=0.5 \mathrm{GeV}^{2}$; solid line-for $Q^{2}=0.5 \mathrm{GeV}^{2}$; dot-dashed line-for $Q^{2}=5 \mathrm{GeV}^{2}$; dashed line-for $Q^{2}=10 \mathrm{GeV}^{2}$.

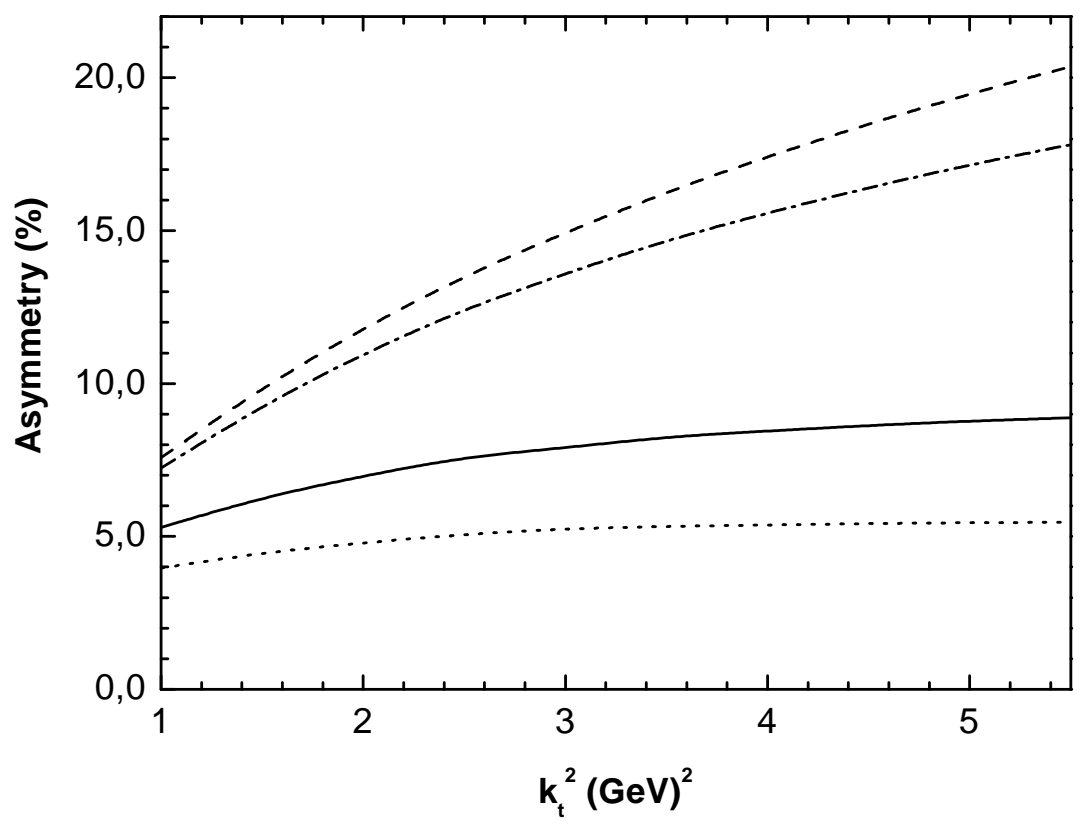

Figure 9: The $A_{l T}^{k}$ asymmetry in diffractive heavy $Q \bar{Q}$ production at $\sqrt{s}=20 \mathrm{GeV}$ for $x_{P}=0.1, y=0.5,|t|=0.3 \mathrm{GeV}^{2}$ : dotted line-for $Q^{2}=0.5 \mathrm{GeV}^{2}$; solid line-for $Q^{2}=$ $0.5 \mathrm{GeV}^{2}$; dot-dashed line-for $Q^{2}=5 \mathrm{GeV}^{2}$; dashed line-for $Q^{2}=10 \mathrm{GeV}^{2}$. 


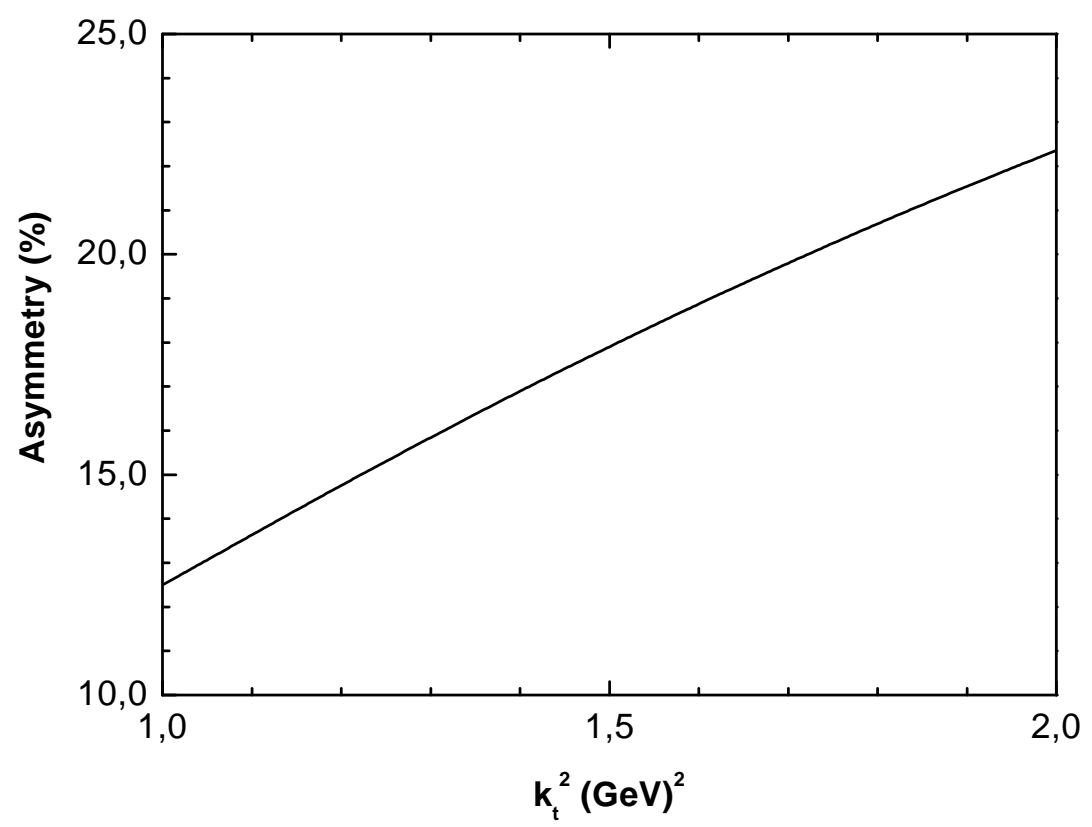

Figure 10: The $A_{l T}^{k}$ asymmetry in diffractive light $Q \bar{Q}$ production for $Q^{2}=5 \mathrm{GeV}^{2}$, $x_{P}=0.1, y=0.5,|t|=0.3(\mathrm{GeV})^{2}$ at $\sqrt{s}=7 \mathrm{GeV}$.

to emphasize here that to observe this contribution to asymmetry, it is necessary to distinguish experimentally the quark and antiquark jets. This can be realized presumably by the charge of the leading particles in the jet which should be connected in charge with the quark produced in photon-gluon fusion. This is an indispensable condition in the experimental study of that asymmetry caused by the fact that the transverse momentum of a quark and an antiquark produced in the process are opposite in sign. If we do not separate events with $\vec{k}_{\perp}$ for the quark jet e.g., the resulting asymmetry will be zero.

The spin-dependent cross section vanishes for $Q^{2} \rightarrow 0$, while the spin-average cross section is constant in this limit. As a result, the asymmetry can be estimated as $A_{l T} \propto$ $Q^{2} /\left(Q^{2}+Q_{0}^{2}\right)$ with $Q_{0}^{2} \sim 1 \mathrm{GeV}^{2}$. The $Q^{2}$ dependence of the asymmetry for light quark production at energy $\sqrt{s}=20 \mathrm{GeV}$ is shown in Fig. 8. The predicted asymmetry for heavy $c \bar{c}$ production is approximately of the same order of magnitude (Fig.9).

At the energy $\sqrt{s}=7 \mathrm{GeV}$ (HERMES) it is not so easy to study the perturbative region for $Q \bar{Q}$ production. Really, $k_{\perp}^{2}$ should be large enough to have a large scale $k_{0}^{2}$ in the process (47). Otherwise, from (41), we have the restriction that $k^{2} \leq M_{X}^{2} / 4$. In this energy range for quite large $M_{X}^{2} \sim(8-10) \mathrm{GeV}^{2} \sim M_{J / \Psi}^{2}$ we find that $\left(k_{\perp}^{2}\right)_{\max } \sim 2 \mathrm{GeV}^{2}$. This means that we can work only in a very limited region of $k^{2}$. The expected $A_{l T}$ asymmetry for light quark production at HERMES is shown in Fig.10. We find that for $k_{\perp}^{2}=1.3 \mathrm{GeV}^{2}, Q^{2}=5 \mathrm{GeV}^{2}, x_{P}=0.1, y=0.5$, and $|t|=0.3 \mathrm{GeV}^{2}$, the coefficient $C_{k}^{Q \bar{Q}}$ in 


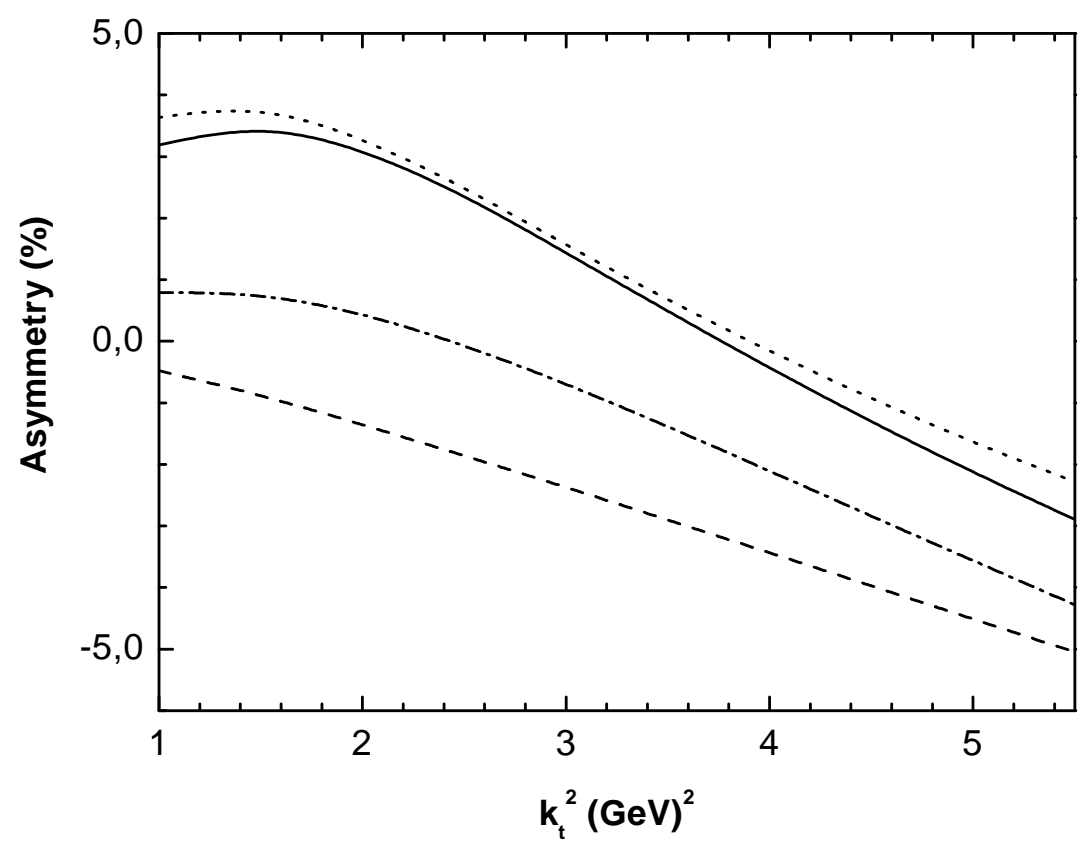

Figure 11: $A_{l T}^{Q}$ asymmetry in diffractive light $Q \bar{Q}$ production at $\sqrt{s}=20 \mathrm{GeV}$ for $x_{P}=0.1$, $y=0.5,|t|=0.3 \mathrm{GeV}^{2}$ : dotted line-for $Q^{2}=0.5 \mathrm{GeV}^{2}$; solid line-for $Q^{2}=0.5 \mathrm{GeV}^{2}$; dotdashed line-for $Q^{2}=5 \mathrm{GeV}^{2}$; dashed line-for $Q^{2}=10 \mathrm{GeV}^{2}$.

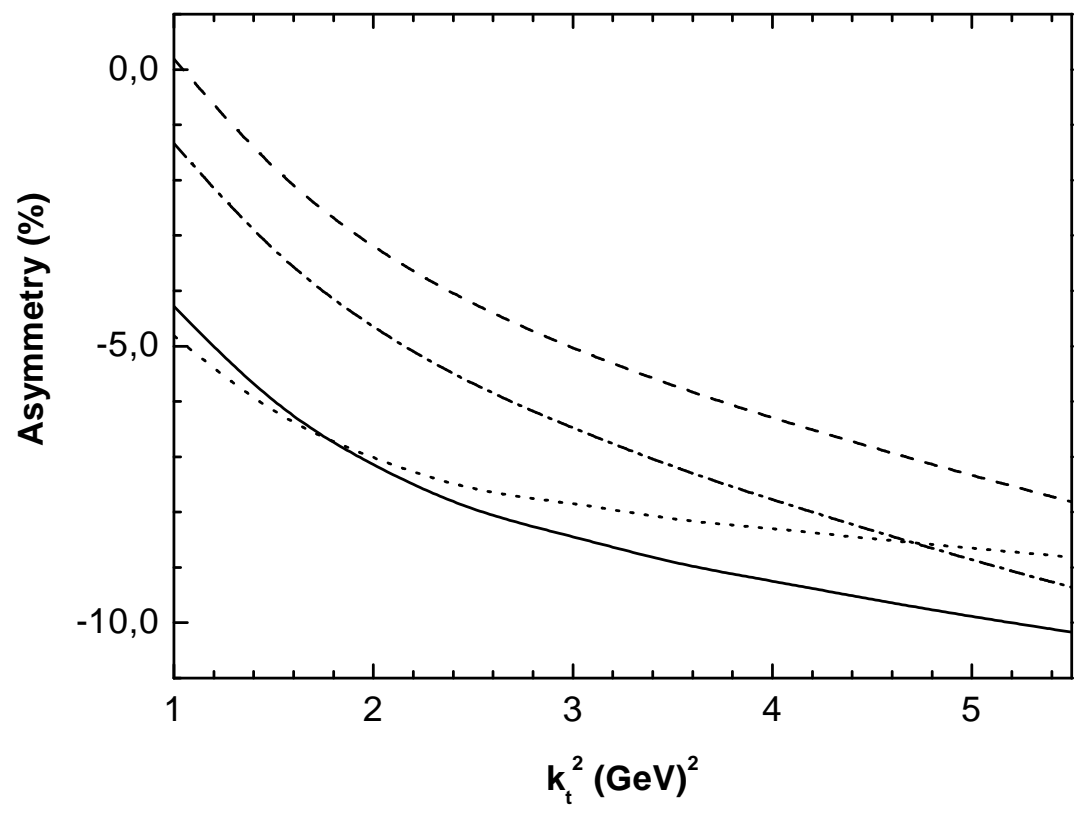

Figure 12: The $A_{l T}^{Q}$ asymmetry in diffractive heavy $Q \bar{Q}$ production at $\sqrt{s}=20 \mathrm{GeV}$ for $x_{P}=0.1, y=0.5,|t|=0.3 \mathrm{GeV}^{2}$ : dotted line-for $Q^{2}=0.5 \mathrm{GeV}^{2}$; solid line-for $Q^{2}=0.5 \mathrm{GeV}^{2}$; dot-dashed line-for $Q^{2}=5 \mathrm{GeV}^{2}$; dashed line-for $Q^{2}=10 \mathrm{GeV}^{2}$. 


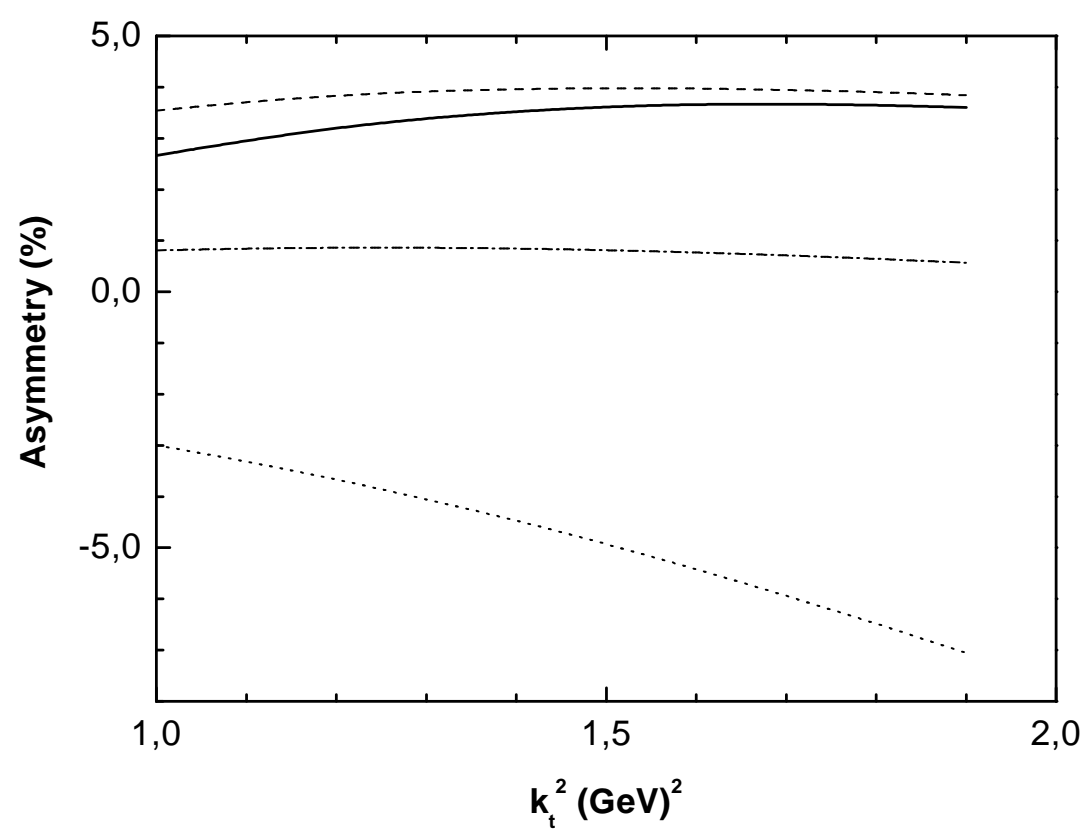

Figure 13: The The $A_{l T}^{Q}$ asymmetry in diffractive light $Q \bar{Q}$ production at $\sqrt{s}=7 \mathrm{GeV}$ for $Q^{2}=5 \mathrm{GeV}^{2}, x_{P}=0.1, y=0.5$ : dotted line -at $|t|=0.1 \mathrm{GeV}^{2}$; dot-dashed line -at $|t|=0.3 \mathrm{GeV}^{2}$; dashed line -at $|t|=0.5 \mathrm{GeV}^{2}$; solid line- integrated over $|t|$ asymmetry.

(58) is quite large, about 1.5 at the HERMES energy. This shows a possibility to study the polarized gluon distribution $\mathcal{K}_{\zeta}^{g}(x)$ in the HERMES experiment.

The contribution to asymmetry $\propto \vec{Q} \vec{S}_{\perp}$ is simpler to study experimentally. Moreover, this term is connected directly with the diffractive contribution with the $A_{\perp}$ asymmetry [27]. We shall analyze this term for the case when the transverse jet momentum $\vec{Q}_{\perp}$ is parallel to the target polarization $\vec{S}_{\perp}$ (a maximal contribution to the asymmetry). The predicted $A_{l T}^{Q}$ asymmetry in diffractive light $Q \bar{Q}$ production at $\sqrt{s}=20 \mathrm{GeV}$ is shown in Fig. 11. This asymmetry is not small for $Q^{2} \sim(0.5-1) \mathrm{GeV}^{2}$. In contrast to the $A_{l T}^{k}$ term, the $A_{l T}^{Q}$ asymmetry has a strong mass dependence. For heavy quark production, this asymmetry becomes negative, Fig. 12 .

It is interesting to look for what we expect to observe for light quark production at low energy $\sqrt{s}=7 \mathrm{GeV}$. The predicted asymmetry for different momentum transfers is shown in Fig. 13. Note that in fixed-target experiments, it is usually difficult to detect the final hadron and determine the momentum transfer. In this case, it will be good to have predictions for the asymmetry integrated over momentum transfer

$$
\bar{A}_{l T}^{Q}=\frac{\int_{t_{\min }}^{t_{\max }} \sigma(-) d t}{\int_{t_{\min }}^{t_{\max }} \sigma(+) d t}
$$

We integrate cross sections from $t_{\min } \sim\left(x_{P} m\right)^{2} \sim 0$ up to $t_{\max }=4 \mathrm{GeV}^{2}$. The predicted 
integrated asymmetry (see Fig. 13) is not small, about $3 \%$.

\section{Conclusion}

In the present paper, diffractive hadron leptoproduction for a longitudinally polarized lepton and a transversely polarized proton at high energies has been studied within the two-gluon exchange model. The polarized cross sections of diffractive hadron production are determined in terms of the leptonic and hadronic tensors and the squared amplitude of hadron production through the photon-two-gluon fusion. The hadronic tensor is expressed in terms of the two-gluon couplings with the proton that are related to SPD. As a result, the cross sections of diffractive meson and $Q \bar{Q}$ production are expressed in terms of the same integrals which are connected with the gluon $\operatorname{SPD} \mathcal{F}_{\zeta}(x)$ and $\mathcal{K}_{\zeta}(x)$.

The $A_{l T}$ asymmetry is found to be proportional to the ratio of $\mathcal{K} / \mathcal{F}$ structure functions and generally can be used to get information on the transverse distribution $\mathcal{K}_{x_{P}}^{g}\left(x_{P}, t\right)$ from experiment. The asymmetry of vector meson production is expected to be quite small, $A_{l T}<0.1 \%$ in the HERMES energy range. This result was obtained for a simple nonrelativistic form of the vector meson wave function and generally can be used only for heavy meson production. The $A_{l T}$ asymmetry for $Q \bar{Q}$ production contains two independent terms which are proportional to the scalar products $\vec{k}_{\perp} \vec{S}_{\perp}$ and $\vec{Q} \vec{S}_{\perp}$ (50). The first one $\propto \vec{k}_{\perp} \vec{S}_{\perp}$ have no $x_{P}$ suppression and is predicted to be about $10 \%$. It might be an excellent object to study transverse effects in the proton coupling with gluons. However, the experimental study of this asymmetry is not so simple. To find nonzero asymmetry in this case, it is necessary to distinguish quark and antiquark jets and to have a possibility to study the azimuthal asymmetry structure. This is an important condition, because the polarized cross section integrated over $d \phi_{J e t}$ is equal to zero. Note that the asymmetry of the same order of magnitude was predicted for diffractive $Q \bar{Q}$ production in polarized proton- proton interaction [41].

The second term $\propto \vec{Q} \vec{S}_{\perp}$ in the $A_{l T}$ asymmetry of $Q \bar{Q}$ production is related to the diffractive contribution to the $A_{\perp}$ asymmetry. The expected asymmetry in this case is not

small too. The predicted coefficient $C_{Q}^{Q \bar{Q}}$ in (58) is about 0.3. This shows the possibility to study the ratio of polarized gluon distributions $\mathcal{K} / \mathcal{F}$ in diffractive $Q \bar{Q}$ leptoproduction. All these results should be applicable to the reactions with heavy quarks. For processes with light quarks, our predictions can be used in the small $x$ region $(x \leq 0.1$ e.g.) where the contribution of quark SPD is expected to be small.

Now, we have no the definite predictions for the $A_{l T}$ asymmetry in light vector meson 
production. Including the transverse quark motion and higher twist effects for transversely polarized $\rho$ meson might be important in asymmetry. In the region of not small $x \geq 0.1$ in the HERMES experiments, the polarized quark SPD might be studied together with the gluon distribution in the case of $\rho$ production. In the case of $\phi$ production, the strange quark SPD might be analyzed. Similar experiments can be conducted at the future COMPASS spectrometer if a transversely polarized target is constructed there.

We conclude that important information on the spin-dependent SPD at small $x$ can be obtained from the asymmetries in diffractive hadron leptoproduction for longitudinally polarized lepton and transversely polarized hadron targets.

We would like to thank A. Borissov, A. Efremov, P. Kroll, T. Morii, O. Nachtmann, W.-D. Nowak, and O. Teryaev for fruitful discussions.

This work was supported in part by the Russian Foundation for Basic Research, Grant 00-02-16696.

\section{References}

[1] A.V. Radyushkin, Phys.Rev, D 56, 5524 (1997).

[2] X. Ji, Phys.Rev. D 557114 (1997).

[3] J.C. Collins, L. Frenkfurt, M. Strikman, Phys. Rev. D56, 2982 (1997).

[4] F.E. Low, Phys. Rev. D12,163 (1975)

S. Nussinov, Phys. Rev. Lett. 34, 1286 (1975).

[5] ZEUS Collab., J. Breitweg et al., Z. Phys, C75, 215 (1997).

[6] H1 Collaboration, S. Aid et al., Nucl. Phys. B472, 3 (1996).

[7] H1 Collab., C. Adloff et al., Eur. Phys. J. C10, 373 (1999).

[8] ZEUS Collab., J. Breitweg et al., Eur. Phys. J. C5, 41 (1998);

H1 Collab., C. Adloff et al., Eur. Phys. J. C6 421 (1999).

[9] HERMES Collab., A. Airapetian et al, Phys. Lett. B513, 301 (2001). 
[10] M.G. Ryskin, Z. Phys. C57, 89 (1993).

[11] S.J. Brodsky at al., Phys. Rev. D50, 3134 (1994).

[12] B. Clerbaux, Elastic production of Vector Mesons at HERA: study of the scale of the interaction and measurement of the helicity amplitudes. E-print: hep-ph/9908519.

[13] M.G. Ryskin, R.G. Roberts, A.D. Martin, E.M. Levin, Z. Phys. C76, 231 (1997).

[14] J.L. Cudell, I. Royen, Nucl. Phys. B545, 505 (1999).

[15] D.Y. Ivanov, R. Kirshner, Phys. Rev. D58, 114026 (1998).

[16] L. Mankiewicz, G. Piller, T. Weigl, Eur. Phys. J. C5, 119 (1998).

[17] H.W. Huang, P. Kroll, Eur. Phys. J. C17, 423 (2000).

[18] M. Vänttinen, L. Mankiewicz, Phys.Lett. B434, 141 (1998).

[19] L. Mankiewicz, G. Piller, Phys. Rev. D61, 074013 (2000).

[20] M.Diehl, Z. Phys. C66, 181 (1995).

[21] J. Bartels, C. Ewerz, H. Lotter, M.Wüsthoff, Phys. Lett. B386, 389 (1996).

[22] E.M. Levin, A.D. Martin, M.G. Ryskin, T. Teubner, Z. Phys. C74, 671 (1997).

[23] B. Lehmann-Dronke, M. Maul, S. Schaefer, E.Stein, A. Schäfer, Phys.Lett. B457, 207 (1999).

[24] J. Bartels, T. Gehrmann, M.G. Ryskin, Eur. Phys. J. C11, 325 (1999).

[25] Proceedings of the Topical Workshop "Transverse Spin Physics", DESY Zeuthen, July 2001, editted by: J. Blümlein, W.-D. Nowak, G. Schnell, Internal Report DESY Zeuthen 01-01 August 2001.

[26] S.V. Goloskokov, Spin effects in diffractive hadron photoproduction. Proc. of the 14th International Spin Physics Symposium, SPIN2000, editted by: K. Hatanaka, T. Nakano, K. Imai, H. Ejiri. AIP Conference Proc. V.570, 541; hep-ph/0011341; hep-ph/0110212.

[27] M. Anselmino, A. Efremov, E. Leader, Phys. Rept. 261, 1 (1995).

[28] M. Anselmino, P. Kroll, B. Pire, Z. Phys. C36, 36 (1987). 
[29] L.V. Gribov, E.M. Levin, M.G. Ryskin, Phys. Rept. 100, 151 (1983).

[30] S.V. Goloskokov, P. Kroll, Phys. Rev. D 60, 014019 (1999).

[31] S.V. Goloskokov, Phys.Lett. B315, 459 (1993).

[32] S.V. Goloskokov, S.P. Kuleshov, O.V. Selyugin, Z. Phys. C50, 455 (1991).

[33] D.C. Peaslee et al., Phys. Rev. Lett. 51, 2359 (1983).

[34] N. Akchurin, S.V. Goloskokov, O.V. Selyugin, Int.J.Mod.Phys. A14, 253 (1999).

[35] E.L. Berger, D. Jones, Phys. Rev. D23, 1521 (1981).

[36] S.V. Goloskokov, Eur. Phys. J. C11, 309 (1999).

[37] S.V. Goloskokov, On the $\sigma_{L} / \sigma_{T}$ ratio in polarized vector meson photoproduction. Proc. of XV International Seminar on High Energy Physics Problems "Relativistic Nuclear Physics and Quantum Chromodynamics", Dubna September 25-29, 2000; hep-ph/0012307.

[38] A. Donnachie, P.V. Landshoff, Nucl. Phys. B244, 322 (1984).

[39] T. Arens, M. Diehl, O. Nachtmann, P.V. Landshoff, Z. Phys. C74, 651 (1997).

[40] ZEUS Collab., J. Breitweg et al., Eur. Phys. J. C14, 213 (2000).

[41] S.V.Goloskokov, Phys.Rev. D53, 5995 (1996). 